\title{
How Best You Can Utilize Your Computer to Tackle Engineering Problems
}

\author{
Sara I. Khaddaj, Dima A. Fares, M. Khaled Joujou, Karim Y. Kabalan, and Ayman Kayssi
}

\begin{abstract}
This paper presents an educational experience on how best engineering students can utilize their computers to tackle engineering problems. The study is matured from a long teaching experience of the Introduction to Electrical and Computer Engineering course required from all first year students majoring either in Computer and Communications Engineering (CCE) or in Electrical and Computer Engineering (ECE) at the Electrical and Computer Engineering Department of the American University of Beirut (AUB). In addition to raising awareness of the engineering topics, the course focuses on the innovative approach of using software tools in considering real-life problem solutions in order to adopt them to new educational technology. This has been achieved by carefully designed experimental sessions where software tools are introduced with an objective of illustrating the technical and analytical computing environment such as Microsoft Office, PSPICE, and MATLAB. These tools are integrated, in this setting, for students to visualize programming environment in solving and documenting engineering problems. In addition the students are introduced to different information research tools to better enhance and support their problem solving skills by using proper referencing.
\end{abstract}

Index Terms-Electrical engineering, introduction to engineering, LABVIEW, MATLAB, moodle, PSPICE, undergraduate engineering studies.

\section{INTRODUCTION}

For so many years, the department of Electrical and Computer Engineering at the American University of Beirut introduced a new course to first year engineering students on Introduction to Electrical and Computer Engineering. Parts of the main objectives of this course were to introduce students to engineering profession and to some basic mathematical and computing tools used in electrical and computer engineering in addition to fostering communication and teamwork skills. The course contents and approaches have changed over the years based on continuous assessment and feedback obtained from senior students and faculty members.

Many papers have been published on this topic including course objectives, content, teaching approach, and design project. The authors of [1], [2] addressed the need to introduce a similar course in an effort to help students in selecting a major as early as possible of their study and informing them of their educational programs and career

Manuscript received October 4, 2013; revised December 18, 2013.

S. I. Khaddaj, D. A. Fares, M. K. Joujou, K. Y. Kabalan, and A. I. Kayssi are with the Department of Electrical and Computer Engineering, American University of Beirut, Beirut, Lebanon (e-mail: sk56@aub.edu.lb, daf03@aub.edu.lb, mj07@aub.edu.lb, kabalan@aub.edu.lb, and ayman@aub.edu.lb). opportunities. Others [3]-[5] addressed the need of introducing design projects at an early stage in engineering studies to help students to explore design alternatives, to learn design concepts, to solve facing challenges, and to communicate their design solutions. In other cases, the course is meant to strengthen the fundamental problem solving skills, highlight the various engineering disciplines, explore the different areas of electrical engineering with hands on approach, and prepare them to have ingenuity and creativity [6]-[8]. In other related publications, "teaching-in-context" approach is used [9] and others showed the positive background and the obstacles faced during the course's progression, relating all different experiences, from Hands-on to Problem Based Learning [10].

The Course under discussion addresses most of the ideas elaborated before and concentrates on many of the topics addressed in electrical engineering. In addition to introducing students to other engineering majors and to the different areas in the program, the students work in group on multi-disciplinary projects assigned to them earlier in the semester to foster the design, group work, and communication skills. Other important component of this course, which is illustrated in the paper, is the use of software tools in solving and presenting engineering problems as students will appreciate the best use of their computers. This part of the course focuses on Microsoft office tools for communication skills, integrating MATLAB and PSPICE in solving engineering problems [11], [12] so as to make students appreciate and learn and appreciate the use of these packages, and PSPICE usages in electric circuits as documented in many other publications [13]-[15] as well as using information resources.

This paper describes carefully designed experiments where software tools are introduced with an objective of illustrating the technical and analytical computing environment such as Microsoft Office, PSPICE, and MATLAB. These tools are integrated, in this setting, for students to visualize programming environment in solving and documenting engineering problems. Moreover, students comprehend the use of online and offline library resources for fast and impeccable search of information.

\section{COMPUTER LAB OVERVIEW}

The 3-Credit Introduction to Electrical and Computer Engineering course offered at AUB consists of two weekly lectures and a weekly 3-hour lab session over a period of 10 weeks. The laboratory component is split into two main categories; the hardware-based experiments and the computer or software-based experiments. The five 
hardware-based experiments, implemented using NI Lab VIEW and a DSP hardware kit, cover fundamental engineering applications such as signal processing, robotics, communication, computer vision, and digital image processing. In addition to the hardware-based experiments, a course design project is introduced whose objective is to assist student to become proficient in using their hardware and software skills to build a functional model and control it [16]. The five computer-based experiments, subject of this paper, are intended to introduce students to different software packages and tools that are useful for their engineering studies.

While during the hardware-laboratory sessions and project students work as teams of two to four students to foster teamwork skills, students in the software laboratory sessions work individually to give each student the chance to be self-dependent and self-innovators, and to insure individual competence in learned software tools. To fulfill these objectives, registered students are split into different computer laboratory rooms; each equipped with 20 computer stations, and are supervised by experienced graduate students under the direction and follow-up of the course instructor. The rationale behind having TAs teach those labs is to have a good instructor to student ratio while sparing department resources (faculty and time) to more advanced components of this course (hardware-based experiments, project, lectures, ...) and to provide the graduate students with a teaching experience throughout their graduate studies at the university.

In addition to an introductory session, the remaining experiments cover Microsoft Office (Excel/Word), introduction to MATLAB, introduction to Pspice, SPICE/ MATLAB application on engineering problems, and library resources and information sources usage.

\section{A. The Introductory Lab Session}

In the introductory session, students are introduced to the lab objectives, logistics, lab environment, assessment tools as well as e-learning tools that will introduce them to Moodle learning environment. Familiarizing students with Moodle software platform is essential since all course material and lab manuals are posted on Moodle. Besides, news forums are created on Moodle course page to facilitate the instructor(s) communication with students and send them relevant course announcements when needed. Discussion Forums are also used to provide students with a space where they can interact with their peers and instructors especially in the course project execution phases. The course also relies on Moodle activities such as students' reports submissions as well as on-line quizzes and assignments used for course assessment.

\section{B. The Microsoft Office Excel/Word Session}

In the Microsoft Office Excel/Word session, first year engineering students come from different high schools with different backgrounds and levels in computer usage. Although all students know how to use Microsoft Office Word and Excel programs, it is observed that most of them lack the knowledge of very important available functionalities and tools in these programs. To solve this problem and unify students' knowledge levels, an experiment introducing students to important tools relevant to engineering studies are introduced. In this experiment, students are asked to formulate and solve mathematical models using a spreadsheet and learn how to write the mathematical formulation using the Word AddIn "Mathtype". They study how to edit and display the results using tables and charts. After completing the assignment, students are asked to prepare a report that explains the work done during the session. This session empowers students with the basic usage of spreadsheets and the technicality of writing reports on Microsoft Office Word.

At the beginning of the assignment, students are introduced to Excel main window. They comprehend the location of the title bar, function bar, name box, status bar, and other functions. A problem is introduced to make it easier for students to incorporate the modeling procedure in excel. The problem addresses a recent engineering topic that is of interest for first year students which is renewable energy resources. Mainly, the instructor explains to the students some of these resources such as the wind turbines and solar cells. Then, a quick description of wind turbine systems is put forth. Students comprehend the modeling of wind turbines and the extraction of the power $(\mathrm{P})$ obtained from a wind turbine having a certain radius $(\mathrm{R})$ and at a certain wind speed (v) by varying the wind speed and area of the wind turbine. Using the spreadsheet, they take incremental wind speed values while fixing the turbine radius and calculate the power output of the turbine. Then, they calculate the output power while fixing the wind speed and using incremental radius values. The students use a chart to comprehend the effect of wind speed and area of wind turbine on the output power of the wind turbine. After the session ends, and in addition to getting familiar with excel basic window, students learn some of excel functionality such as: performing mathematical operations on cells, fixing a certain cell, inserting charts, and others.

\section{The Matlab Session}

The Matlab session introduces students to Matlab as a helpful mathematical tool used by engineering students in many courses offered by the department. For this reason, it is important to expose first year engineers to this software tool so that it will be easier for them at later stages to customize this tool for their needs. During the assignment, the instructor explains some of Matlab syntaxes. First, the way Matlab understands polynomials as a vector and some of the Matlab functions used in polynomial computations such as: polynomial addition and subtraction, polynomial multiplication and division using Conv and Deconv functions, respectively, and finding the roots of a polynomial using Matlab function "roots". Next, students learn how to integrate, differentiate and solve differential equations using Matlab functions int, diff, and dsolve. In addition to that students solve three equations with three unknowns using the solve function. Then, the instructor formulates for them the problem in a different way by writing the equations in a matrix format as $\mathrm{Ax}=\mathrm{B}$ and solving them using the Matlab backslash operator (A\B). Students are then asked to verify that the results from both methods match. The instructor highlights the difference between the backslash operator and the division sign. The instructor reveals that behind this backslash operator is a deep program based on newton's 
method and other optimization techniques. In addition to matrix manipulations, students also comprehend the syntax of complex number using Matlab by calculating the angle and magnitude of a complex number as well as writing a complex number using different formats. Finally, students acquire plotting data points and functions using Matlab. The assignment incorporates a diversity of functions that is used in Matlab and needed by engineering students in their upcoming courses.

\section{The Pspice Session}

A Pspice section is this course is introduced as it is heavily used as a simulator tool in all introductory courses in circuits and electronics offered by the department. Hence, this laboratory session introduces students to Pspice as a powerful general purpose analog circuit simulator used to verify circuit designs and predict circuit behavior especially in complex circuit problems.

In this experiment, students are introduced to the main features of Pspice along with the technicality of building a circuit and simulating the output at desired nodes. Then, they build, simulate and analyze three different circuit problems that they usually encounter in their courses. The first problem is a resistive circuit with a voltage source and two resistors. It is simulated using bias point analysis and results are verified against their manually calculated ones. Then, students use DC sweep analysis to simulate the output for different voltage levels. The second problem addresses a time domain analysis using an RC circuit with a pulsed voltage source. They learn the use of parametric sweep tool to simulate the output for different resistors levels. The third problem tackles AC sweep analysis using an LC filter circuitry. Through this problem, students learn the difference between time and frequency domains. They perform transient analysis on the output voltage to analyze the effect of the filter. The assignment surfaces the main tools into Pspice analysis which equip first year engineering students with this tool for analysis.

\section{E. The Spice/Matlab Session}

The SPICE/MATLAB application on engineering problems session was introduced in 2009 to replace an experiment introducing Xilinx ISE software for digital design. The change was due to a survey conducted by the department as part of continuous assessment of the course. Survey results showed that the majority consider this experiment as heavy and complicated. On the other hand, more than $60 \%$ of the students felt that more programming labs (Especially MATLAB and PSPICE) were very important and very useful due to their practical use in other courses and allocating more time for those experiments is helpful early in first year. Accordingly, course instructors tried to develop a new experiment based on these software tools to reinforce them as technical computing environments used to solve practical engineering problems. Accordingly, an innovative approach was introduced in the developed experiment by finding a correlation between the MATLAB and PSPICE tools and applying the acquired knowledge to solve Electric Circuits problems [17]. The idea is based on considering a circuit problem, analyze using learned techniques, drive circuit equations, solve them using Matlab, and finally compare results using Pspice. The experiment includes different selected circuitry problems that are usually encountered by students in basic electric circuit courses for electrical and computer engineers. To analyze each problem, a systematic three-level approach is developed:

1) Theoretical analysis: The first level includes providing a circuit diagram showing an electrical design that aims to meet specific learning topics from introductory circuit courses. The schematic is provided to the students prior to the lab experiment for them to analyze. Using the acquired knowledge of circuit analysis techniques, they solve the circuit problem analytically, find some parameters, and interpret their calculations.

2) MATLAB: The second level includes using learned MATLAB analytical tools learned in the previous experiment to solve mathematical equations derived from theoretical analysis conducted. Students will appreciate using MATLAB tools and functions to reduce computational complexity in advanced or complex circuits.

3) PSPICE: The third level includes using SPICE analysis tools learned in previous experiment to simulate and solve electric circuits' problems directly from the schematic. To close the loop, students are asked to validate the obtained theoretical results with the simulation results.

The three-level approach insures student comprehension of different circuit analysis techniques and approaches as well as the flexibility in utilizing appropriate software packages, specifically MATLAB and PSPICE tools and functions when needed.

The experiment consists of five electric circuit problems covering classical electric circuits' topics: node voltage and mesh current analysis using independent and dependent sources, step and natural response of RC and RLC circuits, and operational amplifier analysis.

The first circuit consists of a combination of voltage and current independent sources along with resistors which is a typical circuit encountered when solving node voltage analysis. In the first level theoretical analysis, students are asked to write the node voltage equations governing the circuit. Then, they are asked to solve the system of equations using MATLAB commands ('solve') as second-level analysis. Finally, in the third-level, student should draw the schematic using ORCAD capture and properly assign values for circuit variables. The circuit is simulated using DC-bias point analysis after creating a netlist. The importance of a netlist as a link between the circuit diagram and the nodal connection that the machine can understand is highlighted. The significance of correctly placing a ground point and its relevance in circuit analysis is emphasized to students. Finally, students learn how to use voltage markers to simulate the circuit and find the nodal voltages.

The second circuit consists of dependent sources specifically current controlled current dependent source. The instructor explains the four different kinds of dependent controlled sources. In this section of the experiment, students are required to solve the circuit using mesh analysis, use MATLAB to solve the system of equations using the 'Matrix Method', draw the circuit using PSPICE Capture and assign 
the values for all the circuit variables, and simulate the circuit to find the values of the dependent sources.

The third circuit problem is a basic voltage source connected in parallel to a resistor and a capacitor. In this case, the students are requested to derive the first order differential equation of the $\mathrm{RC}$ circuit in terms of $\mathrm{R}$ and $\mathrm{C}$, to determine the solution of this equation in terms of $\mathrm{R}, \mathrm{C}$, and the initial voltage across the capacitor, and to derive the expression of the current flowing through the capacitor in terms of circuit's variables. During the lab session, students are required to use MATLAB to derive the expression of the voltage across the capacitor using 'dsolve' function from the developed equations. They use 'help dsolve' to learn how to input initial conditions. Afterwards, they use MATLAB to find the current expression using the function 'diff' and 'vpa' to get a floating point approximation. On the other hand, students simulate the schematic to plot the waveforms of the voltage and the current of the capacitor using transient analysis simulation. While solving such a problem, students are introduced to using polarized capacitors 'Celec', setting appropriate time window to visualize the response, and understanding the relevance of the time constant $\tau$ and the circuit behavior at $5 \tau$. Students are also implicitly introduced to using the current source 'IPULSE' from the PSPICE library and specifying its properties specifically pulse width and period.

The fourth example covers underdamped natural response of a parallel RLC circuit. In this example, and given the initial current across the inductor and the initial voltage across the capacitor, students develop the second order differential equation of the following second-order RLC circuit, find the characteristic equation of the derived differential equation and calculate the corresponding roots, Neper frequency $\alpha$, and the resonant frequency wo. They are also asked to explain and justify the response type (overdamped, underdamped, or critically damped), and to find the natural response of the voltage taking into consideration the initial conditions. During lab session, the students are required to use MATLAB to find the roots of the characteristic equation of the differential equation using 'roots' function, plot the derived natural voltage response using 'ezplot' as a function of a specific time interval using proper labels of the axes and provide a title to the plot. They are required to find the derivative of the natural response using 'diff' function, the solution of the obtained derivative function using 'solve' function to locate the time at which the natural response takes a maximum point. The obtained point value is substituted in the natural response function, using the natural response function 'inline' and 'feval', to evaluate the value of the function at the obtained value. During PSPICE section, they simulate the schematic to plot the waveform of the voltage across the capacitor. They are instructed on how to use the cursor to find the first maximum of the plot on the waveform graph. There are some remarks students should consider while building the circuit such as knowing the default initial current direction in an inductor.

The fifth example of this experiment covers the operation of an integrating Op-amp amplifier. In this example, students derive the output voltage in terms of the voltage input voltage. Then, using MATLAB, they find the value of the output voltage in the time interval $(0, t)$ seconds using the integration function in MATLAB 'int'. While using PSPICE, students are asked to simulate the schematic to plot the waveform of the output voltage. While solving such a problem, students are introduced to integrate operational amplifiers with resistors and capacitors and set its properties on terms of Vcc.

\section{F. The Library Session}

During the library session, the students are trained on using the university library search engines to search for printed library books, electronic books, electronic references, and encyclopedias. This is a part of the learning process on preparing students to write technical papers and researching the latest literature on a certain topic. The objective is to foster their knowledge in utilizing the different information sources both physical such as library books and documents, as well as electronic resources and subscriptions. In addition to gaining skills in efficient search for information, students will also have to learn how to observe proper citation procedure to avoid any act of plagiarism. This is in line with one of the course outcomes which is "the ability of students to use information technology to recognize and locate reliable sources of information using library databases and web".

A university librarian offers the session to the students. During the session the students are trained on using the university library search engines and are "OLIB search engine" and the different search filters that can be used to narrow down the results. The training also covers the important fields that help the students locate the documents in the library. These fields include classmarks, shelfmarks, availability, and table of contents. In addition to the OLIB database search, students are also introduced to the Knovel search engine. Knovel is a web-based application integrating technical information with analytical and search tools to drive innovation and deliver reliable answer. Following the section on finding books, the students are trained on the proper tools for searching for articles. This is done by using the library tool for locating online and printed journals, and the different search filters available. The training on article finding also covers a wide variety of database such as IEEE Xplore, Inspec, ACM digital library, and Elsevier science direct.

In the last section of the training session, the students are instructed on the importance of proper referencing and the ethical obligation towards intellectual property. They are also trained on how to properly identify if a section or idea needs referencing. Students are also introduced to the different citation styles such as IEEE, APA, and MLA.

\section{COURSE ASSESSMENT}

Students undergo different assessments for each lab session to guarantee that the information required is correctly comprehended. After the Microsoft office session, they start preparing a Post-lab about the assignment where they are required to write a technical report about the formularization, modeling, and implementation of the wind turbine problem using an excel sheet. They include and analyze the results obtained through the use of the spreadsheet as displayed on 
the charts. The format and layout of the technical report is previously explained and documented allowing the students to apply the already learned materials. This helps students to learn from the beginning the mechanism of structuring a technical report required in their engineering upcoming years.

In the Matlab session and the Pspice lab session, a multiple choice Moodle based quiz is used to assess students learning outcome. Results of the two quizzes satisfy the university average requirements. In the Matlab/Pspice session and for every circuit problem, students are asked to document their theoretical analysis, take a clear snap shot of the MATLAB command window showing the written code and the achieved results. Another snapshot of the PSPICE circuit and simulation results including labels, waveform graphs, and cursors values should be captured. Finally, students are then asked to verify that MATLAB and PSPICE results match with the theoretical analysis. A mini report documenting the five problems is submitted by students as in-lab using Moodle as an assessment tool for this lab. Submitted in-labs are corrected and graded and feedback is communicated to students via Moodle.

Moodle is also used during the library session, where the students answer an online quiz in search for different information using the library online database system.

\section{CONCLUSION}

This paper describes designed software based experiments offered in an introductory course in Electrical and Computer Engineering at the American University of Beirut in an effort to help them to tackle, analyze, and document engineering related problems. These tools are integrated, in this setting, for students to visualize programming environment in solving and documenting engineering problems. In addition the students are introduced to different information research tools to better enhance and support their problem solving skills by using proper referencing. Instructors teaching other different courses that utilize the software tools realized a considerable change in the students' readiness and competence in using the software. These observations are in line with the course outcomes and objectives.

\section{REFERENCES}

[1] M. W. Ohland and B. L. Sill, "Communicating the impact of an introduction to engineering course to engineering departments," in Proc. 32nd Annu. Frontiers in Education, Boston, 2002, vol. 2, pp. F3B-17-F3B-20.

[2] R. F. Erlandson, "Rethinking the Introduction to an Engineering Course," IEEE Trans. on Education, vol. 22, no. 4, pp. 161-165, November 1979.

[3] L. George, "Engineering 100: An Introduction to Engineering Systems at the US Air Force Academy," in Proc. 2nd IEEE International Conf. on System of Systems Engineering, San Antonio, 2007, pp. 1-6.

[4] L. Bellamy, B. McNeill, J. Balley, R. Roedel, W. Moor, I. Zwiebel, and D. Laananen, "An introduction to engineering design: teaching the engineering process through teaming and the continuous improvement philosophy," in Proc. 1995 ASEE/IEEE Frontiers in Education Conf., Atlanta, 1995, vol. 2, pp. 4d2.14.

[5] G. C. Beakley and T. W. Price, "Motivating Engineering Freshmen through an Authentic Design Experience," IEEE Trans. on Education, vol. 9, no. 4, pp. 195-197, December 1966.

[6] S. K. Ramesh, "Introduction to Engineering: attract students and strengthen the pipeline," in Proc. 29th Annu. Frontiers in Education Conf., California, 1999, vol. 3.
[7] L. J. Karam and N. Monsef, "Increasing retention through Introduction to Engineering Design," in Proc. 2011 IEEE Digital Signal Processing Workshop and IEEE Signal Processing Education Workshop, Arizona, 2011, pp. 186-191.

[8] R. B. Uribe and L. Haken, "An introduction to electrical and computer engineering for freshmen: laboratories and lectures," in Proc. 24th proceedings of the Frontiers in Education Conf., California, 1994, pp. 68-91.

[9] L. R. Carley, P. Khosla, and R. Unetich, "Teaching "Introduction to electrical and computer engineering" in context," in Proc. the IEEE, vol. 88 , no. 1,2000 , pp. 8-22.

[10] L. M. C. Vilela, M. S. Neto, and R. C. Campos, "Experiences and perspectives of a non-conventional introduction to engineering course," in Proc. 15th International Conf. on Interactive Collaborative Learning, Austria, 2012, pp. 1-2.

[11] L. T. Pillage, "An early introduction to circuit simulation techniques," IEEE Trans. on Education, vol. 36, no. 1, pp. 16-19, February 1993.

[12] B. Zoghi, T. C. Lyster, and R. E. Puckett, "Voltage maps simplify electric circuit analysis," in Proc. 1994 IEEE in Southeastcon '94. Creative Technology Transfer-A Global Affair Conf., Florida, 1994, pp. 185-189

[13] M. Zhang, X. Guan, W. Liu, and C. Zhang, "An alternative teaching method in second-order circuit by computer-assisted instruction," in Proc. 4th International Conference on Computer Science \& Education Conf., China, 2009, pp. 1781-1784.

[14] D. W. Hart, "Circuit simulation as an aid in teaching the principles of power electronics," IEEE Trans. on Education, vol. 36, no. 1, pp. 10-16, Febrary 1993.

[15] D. Y. Northam, "Introducing computer tools into a first course in electrical engineering," IEEE Trans. on Education, vol. 38, no. 1, pp. 13-16, February 1995.

[16] S. I. Khaddaj, K. W. Joujou, A. Kayssi, and K. Y. Kabalan, "DSP Experiments and Design Projects in an Introduction to ECE Course at the American University of Beirut," in Proc. 2011 IEEE Digital Signal Processing Workshop and IEEE Signal processing Education Workshop, Arizona, 2011, pp. 95-100.

[17] D. Fares, S. Khaddaj, M. K. Joujou, and K. Y. Kabalan, "A learning Approach to Circuitry Problems Using MatLab and Pspice," in Proc. 2012 IEEE Global Engineering Education Conf. (EDUCON), Morocco, 2012, pp. 1-5.

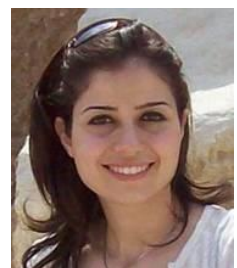

Sara I. Khaddaj was born in Beirut, Lebanon. She received her B.E. and M.E. degree in electrical and computer engineering from the American University of Beirut (AUB) in 2005 and 2007 respectively with distinction. She received Dean's creative achievement award for her undergraduate final project. In 2007, she joined the Department of Electrical and Computer Engineering at AUB as an Instructor and Lab Engineer, where she has developed, coordinated, and taught Introduction to Engineering, Digital and Analog Electronics lab, Computer Organization lab, and Systems Integration Design lab. Her main research interests include engineering education and assessment, information theory and neuroscience. and system identification. She is a member of IEEE, WIE, and Beirut OEA.

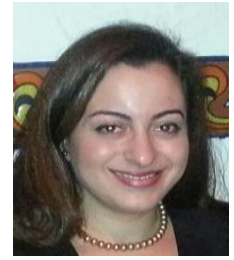

Dima A. Fares was born in Choeuifat, Lebanon. She received the B.S. in electrical engineering from the American University of Beirut in 2003. She received her M.S. degree in electrical and computer Engineering from the American University of Beirut in 2008. She is currently pursuing her $\mathrm{PhD}$ degree from the American University of Beirut. Her main research focus is in the area of Hybrid power systems, renewable energy systems and energy management systems. She is working on a joint project between the American University of Beirut and the University of Applied Sciences in Esslingen Germany.

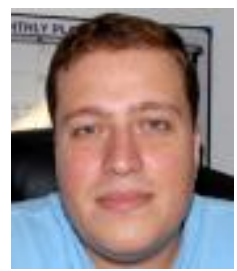

Mohamad Khaled W. Joujou was born in Beirut Lebanon. He received the B.E. degree in electrical engineering and the M.E. in mechatronics from the American University of Beirut (AUB) in 1997 and 2003 respectively. During the period from 1998 till 2003 he was the owner of free-lance company working in the field of industrial automation and a part time instructor at AUB. In 2003 he joined AUB as the laboratory manager for the department of Electrical and Computer Engineering. His main areas of interest are power, electronics, and industrial automation. 


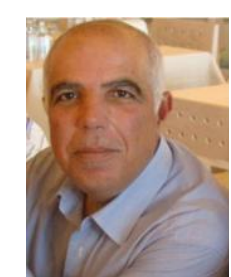

Karim Y. Kabalan was born in Jbeil, Lebanon. He received the B.S. degree in physics from the Lebanese University in 1979, and the M.S. and Ph.D. degrees in electrical and computer engineering from Syracuse University, in 1983 and 1985, respectively. During the 1986 fall semester, he was a visiting assistant professor of Electrical and Computer Engineering at Syracuse University. Currently, he is a professor of Electrical and Computer Engineering with the Electrical and Computer Engineering Department, Faculty of Engineering and Architecture, American University of Beirut. His main research focus is in the area of Electromagnetic and Radio Frequency, microstrip antenna, cognitive radio, and MIMO antenna systems.

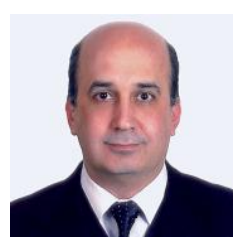

Ayman Kayssi was born in Lebanon. He studied electrical engineering and received the $\mathrm{BE}$ degree, with distinction, in 1987 from the American University of Beirut (AUB), and the MSE and PhD degrees from the University of Michigan, Ann Arbor, in 1989 and 1993, respectively. He received the Academic Excellence Award of the AUB Alumni Association in 1987. In 1993, he joined the Department of Electrical and Computer Engineering (ECE) at AUB, where he is currently a full professor. In 1999-2000, he took a leave of absence and joined Transmog Inc. as chief technology officer. From 2004 to 2007, he served as chairman of the ECE Department at AUB. He teaches courses in electronics and in networking, and has received AUB's Teaching Excellence Award in 2003. His research interests are in information security and networks, and in integrated circuit design and test He has published more than 165 articles in the areas of VLSI, networking, security, and engineering education. He is a senior member of IEEE, and a member of ACM, ISOC, and the Beirut OEA. 